\title{
Terapia Celular na Cardiomiopatia Dilatada
}

\author{
Cell Therapy in Dilated Cardiomyopathy
}

\author{
Helena Furtado Martino, Paulo Sérgio Oliveira, Edmilson Assunção, Rita Villela, Miriam Gaze, \\ Patrícia C. dos Santos Costa, Fernando César Castro Souza, Luiz Henrique Weitzel, \\ Ana Paula R. Velloso, Amarino Oliveira Júnior, Antônio C. Campos de Carvalho \\ Instituto Nacional de Cardiologia Laranjeiras, Hospital Prócardíaco - Rio de Janeiro, RJ
}

\begin{abstract}
Homem de 41 anos em insuficiência cardíaca sistólica, CF III NYHA, estágio clínico C, por cardiomiopatia dilatada, foi submetido ao transplante autólogo da fração mononuclear da medula óssea, via sistema arterial coronariano, através de cateterismo cardíaco. Dois meses após o procedimento, houve diminuição do BNP plasmático, diminuição da área cardíaca ao estudo radiológico do tórax e à ressonância nuclear magnética. O ecocardiograma demonstrou diminuição do fluxo regurgitante secundário a dilatação do anel mitral. Na ergoespirometria houve melhor desempenho, com aumento do consumo máximo de oxigênio, sendo possível redução da terapêutica medicamentosa. A ausência de eventos adversos caracterizados por: instabilidade clínica/hemodinâmica, alteração enzimática ou eletrocardiográfica apontam para segurança e exeqüibilidade deste procedimento realizado e descrito com pioneirismo na cardiomiopatia dilatada.
\end{abstract}

A forty-one-year-old male with systolic heart failure, FC-III NYHA, clinical stage C due to dilated cardiomyopathy was submitted to an autologous transplant of the mononuclear fraction of bone marrow via coronary artery system through heart catheterism. Two months after the procedure, there was a decrease in plasma $B N P$ and cardiac area reduction at the thorax X-ray and nuclear magnetic resonance. The echocardiogram showed decrease of the secondary regurgitation and mitral ring dilatation. There was a better performance at the ergospirometry, with increase of the maximum oxygen consumption and consequent reduction in drug therapy. The absence of adverse events characterized by clinical/hemodynamic instability, enzymatic alteration or electrocardiogram demonstrate the safety and feasibility of this procedure carried out and described with pioneering spirit in dilated cardiomyopathy.

\section{INTRODUÇÃo}

Apesar dos progressos no controle medicamentoso da insuficiência cardíaca sistólica crônica, estágio evolutivo de várias cardiopatias, entre elas a cardiomiopatia dilatada, o número de indivíduos que apresentam a síndrome cresce mundialmente, tornando-a um grave problema de saúde pública. Para um número ainda expressivo de indivíduos, a síndrome progride mesmo com o tratamento medicamentoso. Assim, a insuficiência cardíaca de Classe Funcional III e IV da New York Heart Association tem sido uma das principais causas de internações, consumindo grande parte dos recursos aplicados em saúde e com alta taxa de mortalidade ( $40 \%$ ao ano). 0 desenvolvimento de novos procedimentos terapêuticos de baixo custo e risco, como o implante miocárdico de células-tronco obtidas através de aspirado de medula óssea do próprio indivíduo, constitui promissora opção terapêutica para estas formas avançadas. A partir da década de 90 , inúmeros trabalhos experimentais em modelos animais de lesão cardíaca demonstraram a capacidade de células derivadas da medula óssea em melhorar a performance do coração, quando injetadas diretamente no miocárdio ou na circulação sistêmica. No início do novo milênio, os conhecimentos gerados nos modelos experimentais começaram a ser aplicados na clínica. Em trabalho pioneiro, Menasché e cols. realizaram o transplante de células-satélite de músculo esquelético para o coração de um paciente idoso com insuficiência cardíaca refratária, na França ${ }^{1}$. A seguir, dois grupos na Alemanha e um em Hong-Kong utilizaram células mononucleares de medula óssea no tratamento de pacientes com doença isquêmica ${ }^{2-4}$. No Brasil, no âmbito do projeto do Instituto do Milênio de Bioengenharia Tecidual, foram utilizadas estas mesmas células no tratamento de pacientes com insuficiência cardíaca pós-isquêmica ${ }^{5}$ e mais recentemente em pacientes com cardiopatia chagásica crônica ${ }^{6}$.

\section{Relato do Caso}

Homem de 41 anos, há 12 anos com sintomas progressivos de insuficiência cardíaca sistólica por cardiomiopatia dilatada. Nos últimos dois anos apresentava intensa limitação funcional, cansaço ao tomar banho e episódios de ortopnéia e dispnéia paroxística noturna. Marcadores inflamatórios e provas sorológicas (inclusive para doença de Chagas) negativos. Circulação coronariana livre de lesões obstrutivas à cineangiocoronariografia. 0 paciente encontrava-se com terapêutica otimizada em Clínica de Insuficiência Cardíaca, fazendo uso diário de Digoxina 0,25mg, Furosemida 80mg, Espironolactona $25 \mathrm{mg}$, Captopril $75 \mathrm{mg}$, Carvedilol 50mg. Ao exame físico, o paciente apresentava peso de $66,4 \mathrm{Kg}$, pressão arterial (PA) de 100/60 mmHg, freqüência cardíaca (FC) de 76 bpm, freqüência respiratória (FR) de 20 irpm, ritmo 
cardíaco regular com B3, sopro holossistólico 3+/6+ com irradiação para o dorso, murmúrio vesicular audível sem ruídos adventícios e fígado palpável a $1 \mathrm{~cm}$ do rebordo costal direito, sem edema de membros inferiores. Após informação e consentimento, o paciente foi submetido a: teste de qualidade de vida de Minnesota; radiografia de tórax; dosagem do BNP plasmático, de marcadores inflamatórios e de enzimas séricas; ressonância nuclear magnética; ecocardiograma; eletrocardiograma de 12 derivações; Holter 24 horas; ergoespirometria e estudo hemodinâmico.

Para implante das células no coração, foi aspirado $50 \mathrm{~mL}$ de medula óssea das cristas ilíacas do paciente e separada a fração mononuclear após pré-filtragem

\begin{tabular}{|cccc|}
\multicolumn{4}{|c}{ Tabela 1 - Enzimas séricas } \\
\hline Enzimas & pré & $\mathbf{6 h}$ & $\mathbf{1 2 h}$ \\
\hline CKmb & 13 & 15 & 17 \\
\hline Troponina & 0,5 & 0,5 & 0,5
\end{tabular}

(sistema de filtro de medula óssea da Universidade de Washington - EUA) e centrifugação em gradiente de Ficoll. Após ressuspensão para $20 \mathrm{~mL}$ em soro autólogo $10^{8}$ células foram injetadas no sistema arterial coronariano (10 mL na DA, 4 mL na CX, 6 mL da CD) sem ocorrência de dor, arritmia ou instabilidade hemodinâmica durante o procedimento e no pós imediato.

A monitoração eletrocardiográfica e enzimática foi realizada durante as primeiras 24 horas pósprocedimento, em ambiente de terapia intensiva, não havendo alterações nestes parâmetros (tab. 1).

Setenta e duas horas após o procedimento, o paciente recebeu alta hospitalar, retornando quinzenalmente para consultas de acompanhamento e, ao completar dois meses, os exames descritos acima foram repetidos. Ao exame físico observamos que o paciente manteve-se sem alteração de peso $(66,5 \mathrm{~kg})$, com PA de 100/60 mmHg, FC de 60 bpm, FR de 18 irpm, em ritmo cardíaco regular, mas sem B3, e com redução do sopro holossistólico, agora de $1+/ 6+$ e sem irradiação. O questionário de Minnesota apresentou um escore inicial de 63, tendo diminuído após a terapia celular para 39. A radiografia de tórax revelou um índice cardíaco torácico pré-tratamento de 0,55 e pós-terapia de 0,48 (fig. 1). A ressonância também demonstrou uma diminuição dos volumes sistólico e diastólico de VE, que passaram de 302 mL (VSF) e 371 mL (VDF) pré-tratamento, para 217 e $263 \mathrm{~mL}$, respectivamente, após a terapia celular (fig.2). Entretanto, não houve alteração na fração de ejeção que se

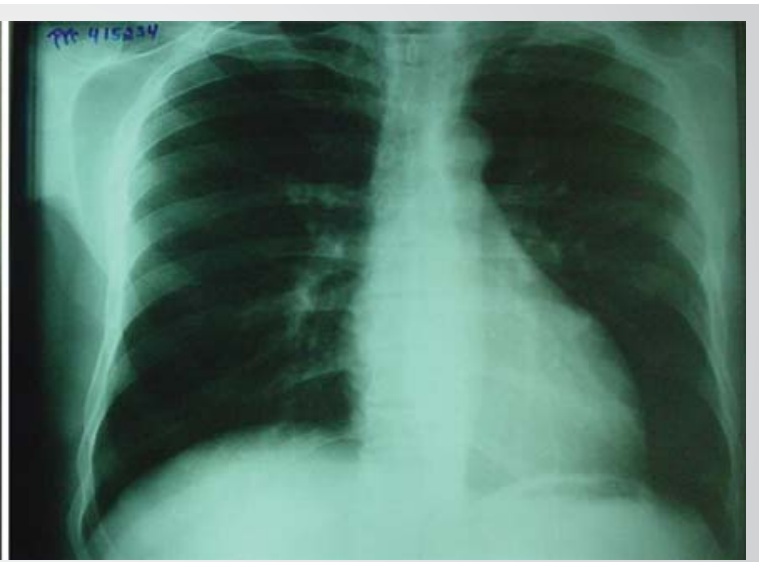

B
A

Fig. 1 - Radiografia do tórax em PA antes (A) e após (B) terapia
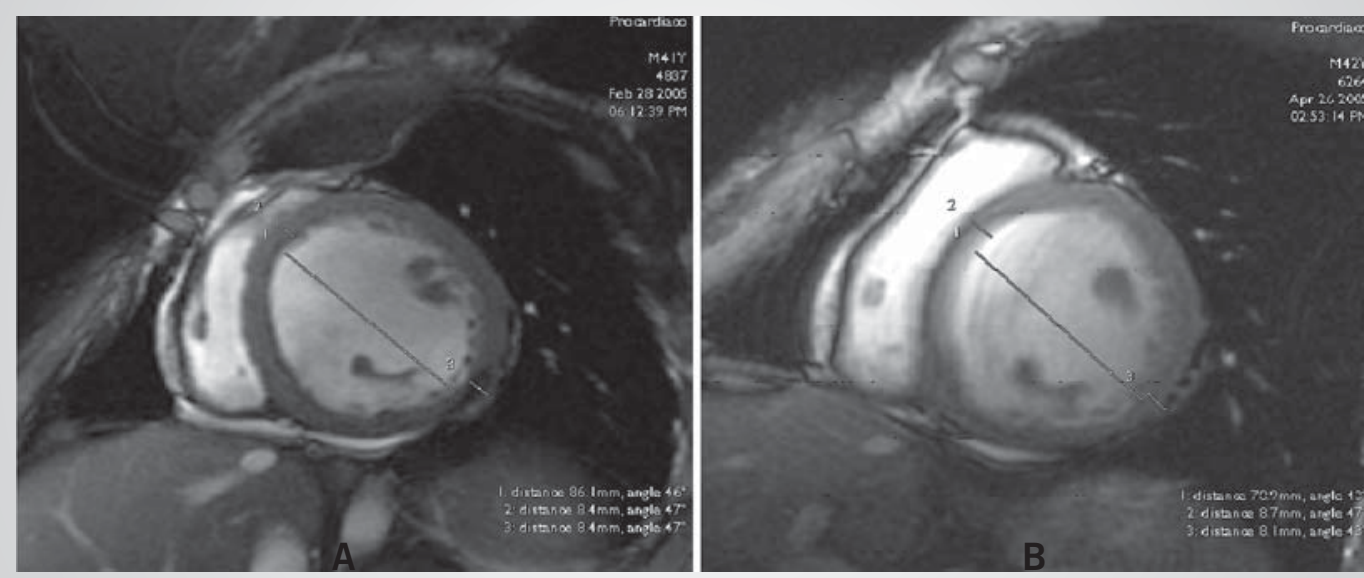
manteve em torno de 17\% (método de Simpson).

Os níveis plasmáticos de BNP variaram de 118 $\mathrm{pg} / \mathrm{mL}$ (pré) para $39 \mathrm{pg} / \mathrm{mL}$ (pós). Ao ecocardiograma observou-se melhora na insuficiência mitral como ilustrado na figura 3.

A melhora observada nas variáveis citadas foi acompanhada por melhor desempenho à ergoespirometria como ilustrado na tabela 2. A destacar que o paciente que se e exeqüibilidade deste novo procedimento.

Em pacientes com cardiopatia chagásica crônica com grave disfunção sistólica, a liberação intracoronariana de células mononucleares de medula óssea (CMMO) também se mostrou segura e resultou em melhora funcional ${ }^{6}$. Com base nestes resultados, utilizamos o modelo de liberação intracoronariana das CMMO em paciente com cardiomiopatia dilatada. No caso

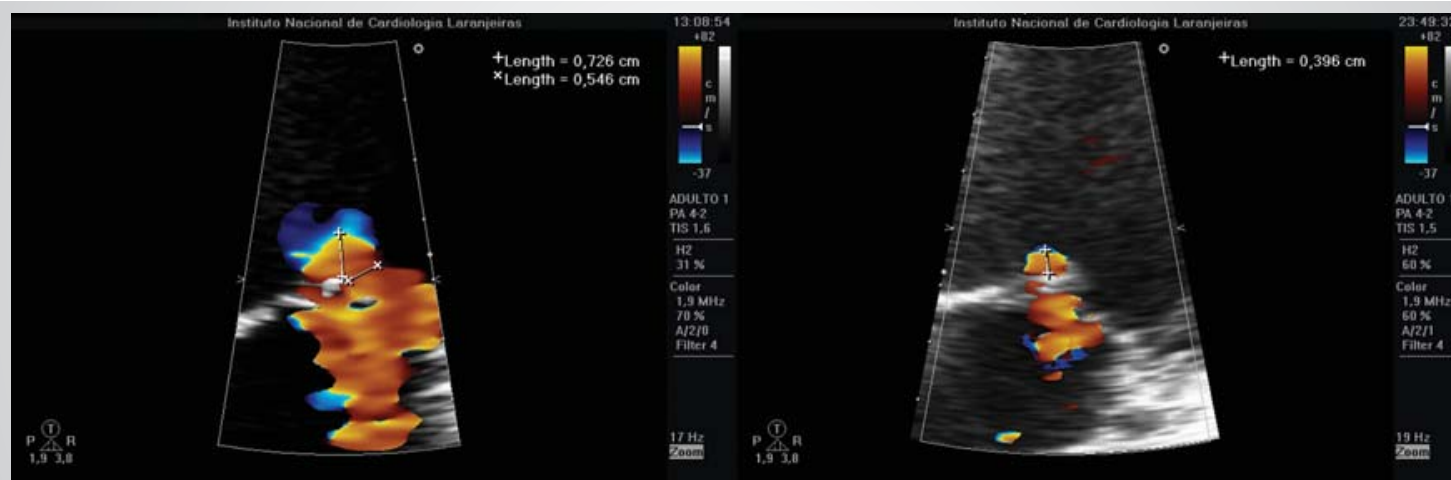

A

B

Fig. 3 - Ecocardiogramas bidimensionais: (A) antes (B) após a terapia, mostrando redução do volume regurgitante mitral de $42 \mathrm{~mL}$ com PISA de 0,726 (A) para $13 \mathrm{~mL}$ com PISA de 0,395 (B)

encontrava em grupo funcional III, por ergoespirometria, antes da terapia, evoluiu para grupo funcional II após o tratamento. A evolução clínica favorável do paciente nos permitiu diminuir a terapêutica medicamentosa com a redução da dosagem diária de furosemida (40mg) e espironolactona (12,5 mg).

\section{Discussão}

A terapia celular tem sido aplicada a pacientes com cardiopatia isquêmica aguda e crônica, através da liberação intracoronariana de células de medula óssea ou de sua injeção intramiocárdica ${ }^{1-5}$. Os mecanismos de ação das CMMO ainda não estão esclarecidos, e há grande controvérsia na literatura sobre sua capacidade de regeneração de cardiomiócitos ${ }^{7-9}$. Ainda assim, em todos os modelos animais de cardiopatia e nos ensaios clínicos até aqui realizados, estas células promoveram melhora de função cardíaca, embora estes estudos tenham sido realizados com o principal intuito de testar a segurança

\section{REFERÊNCIAS}

1. Menasché $P$, Hagege AA, Scorsin M, et al. Myoblast Transplantation for heart failure. Lancet. 2001;357:279-80.

2. Strauer BE, Brehm M, Zeus T, et al. Repair of infarcted myocardium by autologous intracoronary mononuclear bone marrow cell transplantation in humans. Circulation. 2002;106:1913-18.

3. Assmus B, Schächinger V, Teupe C, et al. Transplantation of progenitor cells and regeneration enhancement in acute myocardial infarction (TOPCARE-AMI). Circulation. 2002;106:3009-17.

4. Tse HF, Kwong YL, Chan JKF, et al. Angiogenesis in ischaemic myocardium by intramyocardial autologous bone marrow mononuclear cell implantation. Lancet. 2003;361:47-9.

5. Perin EC, Dohmann HF, Borojevic R, et al .Transendocardial, Autologous Bone Marrow Cell Transplantation for Severe, Chronic
Tabela 2 - Ergoespirometria - Protocolo Rampa

$\begin{array}{lcc}\text { Variável } & \text { Pré } & \text { Pós } \\ \text { Duração } & 5: 49 & 11: 40 \\ \text { Distância (milhas) } & 0,15 & 0,38 \\ \text { FC máx. } & 100 & 102 \\ \text { Pot. Máx. (W) } & 55,9 & 130 \\ \mathrm{VO}_{2} \text { máx. } \mathrm{mL}(\mathrm{Kg} \text {.min) } & 15,61 & 18,64 \\ \text { Grupo funcional } & \text { III } & \text { II } \\ \mathrm{T} 1 / 2 \text { (s) } & >120 & <90 \\ \text { pulso } \mathrm{O}_{2} \text { (mL/bat.) } & 10,3 & 12,1 \\ \mathrm{VE}_{\mathrm{NCO}} & 29,7 & 23,8\end{array}$

relatado, observamos que o procedimento transcorreu sem qualquer intercorrência, sugerindo que o método é seguro e exeqüível. Esta observação aliada à melhora nos parâmetros clínicos e nos exames complementares do paciente é que nos levam a propor a terapia celular como uma alternativa a ser investigada para o tratamento da cardiomiopatia dilatada grave.
Ischemic Heart Failure. Circulation. 2003;107:r75-r83.

6. Vilas-Boas F, Feitosa GS, Soares MPB, et al. Transplante de Células de Medula Óssea para o Miocárdio em Paciente com Insuficiência Cardíaca Secundária a Doença de Chagas. Arq Bras Cardiol. 2004; 82 (2):181-4.

7. Orlic D, Kajstura J, Chimenti S, et al. Bone Marow cells regenerate infarcted myocardium. Nature. 2001; 410: 701-5.

8. Balsam LB, Wagers AJ, Christensen JL, et al Haematopoietic stem cells adopt mature haematopoietic fates in ischaemic myocardium. Nature. 2004; 428: 668-73.

9. Murry CE, Soonpaa MH, Reinecke H, et al. Haematopoietic stem cells do not transdifferentiate into cardiac myocytes in myocardial infarcts. Nature. 2004; 428: 664-8. 